\title{
Finding Email in a Multi-Account, Multi-Device World
}

\author{
Marta E. Cecchinato \\ UCL Interaction Centre \\ University College London \\ WC1E 6BT, London, UK \\ m.cecchinato@cs.ucl.ac.uk
}

\begin{abstract}
Email is far from dead; in fact the volume of messages exchanged daily, the number of accounts per user, and the number of devices on which email is accessed have been constantly growing. Most previous studies on email have focused on management and retrieval behaviour within a single account and on a single device. In this paper, we examine how people retrieve email in today's ecosystem through an in-depth qualitative diary study with 16 participants. We found that personal and work accounts are managed differently, resulting in diverse retrieval strategies: while work accounts are more structured and thus email is retrieved through folders, personal accounts have fewer folders and users rely primarily on the built-in search option. Moreover, retrieval occurs primarily on laptops and PCs compared to smartphones. We explore the reasons, and uncover barriers and workarounds related to managing multiple accounts and devices. Finally, we consider new design possibilities for email clients to better support how email is used today.
\end{abstract}

\section{ACM Classification Keywords}

H.5.m. Information Interfaces and Presentation (e.g. HCI): Miscellaneous

\section{Author Keywords}

Email; multi-device; cross-device interaction; information retrieval; searching; archiving

\section{INTRODUCTION}

Despite the ongoing debate in popular media on whether email is dead ${ }^{1}$, forecasters predict that the number of messages exchanged per year will grow at an average rate of 3\% over the next four years for both business and consumer purposes [19], suggesting that, for the foreseeable future, email is here to stay. This is all the more interesting in the face of the growing popularity of other social media and instant messaging platforms [19], that not only are popular among the younger population ${ }^{2}$,

\footnotetext{
${ }^{1}$ Forbes 2015, http: //onforb.es/1gQ8fXE

${ }^{2}$ Forbes 2014, http://onforb.es/1s5pT1K
}

Permission to make digital or hard copies of all or part of this work for personal or classroom use is granted without fee provided that copies are not made or distributed for profit or commercial advantage and that copies bear this notice and the full citation on the first page. Copyrights for components of this work owned by others than ACM must be honored. Abstracting with credit is permitted. To copy otherwise, or republish, to post on servers or to redistribute to lists, requires prior specific permission and/or a fee. Request permissions from permissions@acm.org.

CHI'16, May 07 - 12, 2016, San Jose, CA, USA.

Copyright (C) 2016 ACM 978-1-4503-3362-7/16/05...\$15.00.

http://dx.doi.org/10.1145/2858036.2858473

\author{
Abigail Sellen, Milad Shokouhi, Gavin Smyth \\ Microsoft Research Cambridge \\ CB1 2FB, Cambridge, UK \\ \{asellen,milads,gavin.smyth\}@microsoft.com
}

but are also starting to be used in the working environment ${ }^{1}$. Alongside its staying power, however, are some of email's equally persistent, attendant problems.

"Email overload", for example, has long been recognised as a universal problem [39] and there is now a substantial body of work dedicated to understanding how people manage and file their email in order to deal with the incoming deluge and growing archives. Over the years, there have been several taxonomies of managing habits (e.g. $[4,17,26]$ ), distinguishing for example between people who frequently, sporadically or never file their email. Regardless of the managing strategy used, there is a trend toward users receiving and retaining more messages [21], reinforcing email's role as an important digital archive. Given this archiving role, understanding how people manage and retrieve email is more important than ever. Previous work has found that how messages are managed is inextricably linked to how users expect to be able to find any given message or set of messages in future [38].

However, the majority of previous studies have overlooked two key factors which have become even more important in recent years: continued growth in the number of accounts per user [19], and the growing number of devices that people use to access their email [30]. Email management across multiple accounts such as work and personal has been largely ignored with only a few exceptions [7, 11, 18]. Email use across devices has only been touched upon as an example of multidevice usage (e.g. [14, 29]), rather than being thoroughly investigated.

We argue that it is now increasingly important to understand user's current email retrieval and management practices across devices and accounts. It may be that the more complex modern ecosystems create new challenges in managing or finding email; it is also possible that having access to multiple devices and accounts have made email management and retrieval easier for some users. Exploring this in depth may point to opportunities for improving email tools. Indeed most have changed very little in the past couple of decades, suggesting they have not kept pace with fundamental changes in the way email is now used.

To this end, we present an in-depth qualitative study with 16 diverse participants who took part in a week-long diary study. We examine their general email habits, their management habits, and how they look for information in their email. Specifically, we investigate how their practices differ across accounts and devices. The results also illustrate issues 
participants encountered during retrieval and workarounds to make finding easier. Consequently, our research confirms and extends previous work in a number of ways and makes the following contributions:

- It further unpacks the differences between work email and personal accounts, with the former relying more on structure and preparatory mechanisms, and the latter relying more on opportunistic retrieval.

- It proves the importance of considering multiple devices when investigating email behaviour. We find how email retrieval occurs primarily on laptops and desktop PCs, and how mobile search is infrequent and often problematic.

- It helps identify current issues with email retrieval and users' workarounds, both in terms of having to manage multiple accounts and deal with device constraints.

- It considers how the design of today's email clients could be improved to better support how email is used in a multiaccount, multi-device ecosystem, and points to trends toward more flexible, integrated tools.

\section{LITERATURE REVIEW}

Most previous research in this area has focused on email as a communication channel at work [37]. Email has also been characterized as a habitat [15] for information management, and as a means of time and task management [26]. As a result, several taxonomies have been created to reflect users' email management strategies, distinguishing between frequent filers, spring cleaners, no filers [39] and few folder filers [17], cleaners and keepers [20], to name but a few. While each of these papers highlights slightly different management strategies, all studied only work-related email.

\section{Email Retrieval}

Managing, filing and archiving work email has been the subject of deeper research than the other side of the coin: retrieving messages in email. Early work, such as Mackay [26], and Whittaker and Sidner [39], distinguished between "preparatory behaviours", where users take time to file and label email in anticipation of retrieving the information, and "opportunistic access", where users rely on sorting, scrolling or searching at the time of need, delaying the burden. However, it was not until later that Whittaker et al.'s work [38] more explicitly showed the relationship between management and retrieval strategies. They compared the benefits of preparatory retrieval using folders and tags with opportunistic search and threading, and showed how these relate to email management strategies. Their findings suggest opportunistic behaviour is better and that preparatory strategies are inefficient and do not necessarily improve successful retrieval. They proposed that filing strategies are a reaction to the large number of messages received and a way to better visualise tasks.

Retrieving email is an intricate research area not only studied in HCI, but also in the information retrieval literature. Of relevance, studies focussed on prototypes called Phlat [13] and Stuff I've Seen [16]. The latter study was aimed at exploring how information is found and re-found on computers, including in email [16]. The system facilitates information re-use, by offering a unified indexing from one's own emails, web history, calendars, and files. The authors found that time frame and people's names are powerful contextual cues used when retrieving personal information, as later reinforced also by Cutrell et al. [12].

\section{Personal vs. Work Email Accounts}

While, on average, people have more than one account to reflect the various facets of one's life [11], most academic research has concentrated on work email to the exclusion of personal email. Smith et al. [34] were the first to point towards a tension between work and personal email, finding in 2003 that $54 \%$ of their participants had two separate accounts, particularly as a way to keep spam out of work email. Ten years later, Capra et al. [7], found that this separation was growing, with $84 \%$ of their survey respondents having separate accounts as a way of managing boundaries between work and personal life. However, it is only more recently that research has shown how work and personal accounts are managed differently. Grevet et al. [18] replicated and extended two seminal studies $[17,39]$ updating our understanding of email management strategies and adding a comparison between work and personal accounts among Gmail-only users. They found that personal inboxes are usually considerably larger in terms of archive size, inbox size and number of unread messages when compared to work inboxes. While these findings are important, more work is needed to unpack personal vs. work use of email in multi-account settings and extending this to other email clients.

\section{Multi-Device Use and Email Access}

Understanding user interactions across multiple devices has emerged as an active area of research in recent years. Takkinen and Shahmeheri [35] were the first to investigate email use on multiple devices exploring how often email is accessed on different computers, and using different email clients as a function of communication context (social, work, educational, etc.) and role. More generally, one's choice of device depends not only on the nature of the task, but also on the context of use (physical space, social environment, etc.) [23, 25], and the access and initialisation time vs. actual usage time [27]. Santosa et al. [31] discussed how email is frequently used to transfer information to others and across devices, especially for quick access, re-access and hot documents [32].

Despite these prior studies, there is relatively little known about cross-device retrieval of email. Montanez et al. [28] investigated multi-device search behaviour in a search log dataset. While they found that there are learnable patterns of device transitions - such as how different devices are used to search different content, and how the choice of device is dependent on the time of the day - their focus is only on browser search and does not mention email use. Carrascal and Church [8] used qualitative and quantitative methods to investigate how mobile search interacts with other mobile apps. They found that "browsers, email, SMS, social networking, shopping \& retail and entertainment related apps are used more intensively when people engage with mobile search, 
both in terms of app launches and duration of app usage" [ibid, p.2745].

\section{Research Questions}

In summary, an examination of related work highlights the need to understand how email retrieval differs between work and personal accounts, especially with respect to multi-device use. Specifically, our work aims to answer the following two research questions:

- What kinds of content do users look for and what strategies do they employ in work vs. personal email accounts for managing and finding email?

- Do users' email retrieval strategies differ across devices? How does this influence users' choice of activity or choice of devices?

\section{METHOD}

In order to get in situ data and maintain high ecological validity, we opted for a mixed methods approach which included a weeklong diary study, with pre- and post-interviews. The study took place in July 2015. Prior studies on email retrieval have relied on observations [14], interviews [29], diary studies [23], and data logging [24]. Our methodology was refined through a series of pilot tests using a diary method which adheres to Carter \& Mankoff's [9] guidelines, asking participants to record instances of retrieval as they occurred, adding more details later if needed and following up with interviews. We defined 'retrieval' for users as a range of behaviours in support of finding information, messages or attachments including: entering keywords in the search bar; accessing one or more folders; glancing or scrolling in folders or inbox; and sorting email based on metadata (date/sender/etc.). Therefore some activities would be more like goal-directed search in pursuit of a particular piece of information (e.g. when using the search bar), whereas other activities such as scrolling or glancing through collections of messages might involve behaviour which would be more like scanning or browsing.

\section{Participants}

Sixteen people completed the study ( 9 women, 7 men), aged between 22 and 52 (Median: 28). All lived in the southeastern area of U.K. Occupations included: a physiotherapist, two nurses, two PhD students, two research assistants, three post-doctoral researchers, a high school teacher, a teaching associate, a barista, a software engineer, a graphic designer, and a marketing coordinator. We screened participants based on occupation, striving to include as many different ones as possible given the initial 35 people who signed up, to ensure a wider spectrum of experiences with email and expertise in computer literacy. Participants were recruited through flyers, social media ads, and word-of-mouth. All participants received an Amazon gift voucher.

\section{Procedure}

Initial Survey

Participants began with a survey covering: general demographics, their email accounts, the total number of folders in both main work and main personal accounts, and their preferred filing strategy for both their main work and main personal accounts (following the classification in [39] of frequent filers, spring cleaners and no filers), the type of devices they owned (including who bought it and where they often used it), and how they accessed their email accounts on each device.

\section{Pre-diary interview}

Participants were asked to bring along devices they used to access email to prompt contextual explanations about their general email habits, including management and retrieval strategies. We asked participants to distinguish between personal and work devices, and accounts when answering questions, collecting pictures of their physical desktops at home and work, and screenshots of their folder structures. The first interview also included observation of retrieval tasks which we assigned in order to create an initial understanding of their practices before the diary study. We asked participants to retrieve messages or information within (e.g. attachments, login details, pictures) from people or services they frequently, rarely, or no longer received emails from. In order to ensure ecological validity, these tasks were based on elements mentioned by themselves during the interview. For example, P12 mentioned going on holiday to Berlin, so we asked him to look for the flight confirmation, as he would normally do. Notes were taken for each step and participants were free to choose which device to use to complete the task.

\section{Diary}

Starting the day after the first interview, participants were then asked to diarise for a week their retrieval activities related to email, ranging from goal-directed search to more general browsing or scanning. We specifically asked them to include, as they occurred, any instances that somehow involved email: whether they were looking for a particular message, or an attachment, or a piece of information within a message, or even if they were just looking through their email in a more serendipitous way. To help them understand what we were interested in, we included examples on each page of the diary. They could choose between a digital $(n=12)$ or paper $(n=4)$ diary, to accommodate their everyday routine. For each entry participants had to specify their location, which device they used and which account they searched. A link to the digital diary was sent out once during the evening of the first interview; reminders to complete the diary were sent daily.

\section{Post-diary interview}

The follow-up interviews were scheduled within a week from the end of the diary, based on participants' availability. The purpose of this interview was to walk the researcher through each diary entry, in order to provide more details and more contextual explanations, building on the in-depth data collection. Both semi-structured interviews took place either in participants' homes, offices, or in our lab. The first interview lasted on average 53 minutes, while the follow-up lasted on average 46 minutes.

\section{Activity logging}

To ensure greater external validity and compensate for underreporting issues in qualitative studies, we asked participants' permission to collect logged data on their usage of email across 
devices, using RescueTime ${ }^{3}$. To make sure participants were comfortable sharing their data, we made this optional, but only six installed it on compatible devices. As a result, logs were only used as an aide-memoir during follow-up interviews, and to validate self-reported data when possible. Before each of the six follow-up interviews, diary entries were compared with logged data to ensure accuracy of self-reported data and we found that no searching activities were missing from the diaries. As previously discussed [3], logs are difficult to collect for different devices due to technical issues (e.g. operating system compatibility) and privacy concerns.

\section{Analysis}

To build a holistic understanding of participants' searching practices across devices and accounts, we compiled interview transcripts, diary entries, notes, photographs, and logged data for the 16 participants, and analysed the data thematically [6]. Open and axial coding was used to define themes, which were then discussed amongst all authors. We looked at differences between work and personal accounts, both in terms of types of email retrieved and strategies used. We then focused the analysis on devices used, and how the type of email, context and affordances defined users' choices. For each stage, we also identified issues and workarounds created by participants, as suggested by [22].

\section{FINDINGS}

Together with the interviews, a total of 239 diary entries was recorded, averaging 15 entries per participant over the course of seven days. In addition, during the first interview, participants completed a total of 42 finding tasks, averaging almost three each. From the initial survey, participants listed having on average three email accounts each (Min: 2, Max: 9, Median: 3). On average people had four devices each (Min: 2, Max: 7, Median: 4), including smartphones, tablets, laptops and desktop PCs, and all participants had devices from a mix of platforms (i.e. Windows, Apple, Android, Linux). Two participants also had a smartwatch: P1 had a Pebble, and P11 had an Apple Watch.

Participants used different email clients, based on device and type of account, i.e. email clients used to access personal and/or work accounts on laptops and PCs were not necessarily the same used on mobile devices. For example, P11 accessed his work and personal accounts on a laptop and PC through webmail (Roundcube and Gmail, respectively), while on his smartphone and tablet he accessed his accounts through the Mailbox app ${ }^{4}$. A list of email clients used across multiple devices and participants is as follows: Work accounts were accessed primarily through Outlook $(\mathrm{n}=15)$, followed by Roundcube ( $n=8)$, Inbox by Gmail $(n=4)$, Gmail $(n=2)$, Mutt $(\mathrm{n}=2)$, Mailbox app $(\mathrm{n}=1)$, Android email app $(\mathrm{n}=1)$, Windows Phone email app $(n=1)$, and finally through K9 app $(n=1)$. Personal accounts, instead, were accessed primarily through Gmail $(n=14)$, followed by Mail app $(n=8)$, Outlook $(n=7)$, Yahoo $(n=3)$, Inbox by Gmail $(n=2)$, Libero $(n=2)$, Mailbox app $(\mathrm{n}=2)$, Mutt $(\mathrm{n}=2)$, and finally the K9 app $(\mathrm{n}=1)$.

\footnotetext{
3 https://www.rescuetime.com

$4_{\text {http: //www . mailboxapp.com }}$
}

We found that reported instances of email retrieval varied by type of account and device used, and also as a function of physical location and activity. Instances were somewhat more frequent for work accounts (55\%) than personal accounts (45\%), loosely overlapping with the location in which these finding activities were conducted: $53 \%$ at work, followed by home (40\%) and other locations (7\%). In terms of devices used, $85 \%$ of finding instances were completed on a laptop $(54 \%)$ or desktop PC $(31 \%)$, followed by the phone $(15 \%)$ and a tablet in just one case, because their "iPhone was on charge, iPad [was the] nearest quickest thing" (P6, diary).

Due to the small sample size and the perhaps low number of self- reported retrieval instances, we need to be cautious in generalising from these numbers, however, the diaries and interviews do give us deeper insights into people's strategies and motivations when looking for email. In the following sections we start by distinguishing between management practices based on different accounts, and then move to what users looked for in work and personal accounts, discussing the why and the how, followed by issues and workarounds. We later discuss how email retrieval differs across devices, based on strategy used, issues encountered and workarounds created when retrieving information across multiple devices.

\section{Email Management in Work vs. Personal Accounts}

All participants had at least one main work-related and one main personal account as a way to maintain separate life roles, and 11 of them had at least one additional account. Only one participant, P10 (who had four email addresses), used her main Gmail account for both personal and work reasons, despite having other work-related accounts. Reasons for having additional accounts confirm those found in Cecchinato et al. [11] (e.g. having legacy accounts, separating one's many life roles, or filtering out junk email), and, if used at all, they were rarely checked or accessed for retrieving email.

The way in which accounts were organised and managed differed between work and personal ones, with work email giving rise to more structured filing. From the initial survey, most participants $(n=10)$ reported having the same overall filing strategy between personal and work accounts: six were frequent filers (P1, P3, P5, P6, P12, P14); two were spring cleaners (P7, P13); and two were no filers (P10, P11). However the remaining six participants $(\mathrm{P} 2, \mathrm{P} 4, \mathrm{P} 8, \mathrm{P} 9, \mathrm{P} 15, \mathrm{P} 16)$ had different strategies for different accounts. Of these, five filed email more frequently in their work account compared to their personal one, while the remaining participant (P4) was more organised in her personal account: this participant, a nurse, reported that she received very few work-related emails relevant to her. Participants tended to have more folders in their work account, suggesting they try to be more organised or used work email more as an archive. P2 described this well: "Somehow I had accepted that I didn't really care that my Gmail [personal] inbox was huge. But $[\cdots]$ I might star things $[\cdots]$. [In] my small inbox at work [Outlook] I labelled [categorised] it and archived it".

These findings are reflected in the average number of folders or labels that participants had in their work and personal accounts (Table 1). Here we treat both folders and labels as equivalent 


\begin{tabular}{lrr} 
& Main work account & Main personal account \\
\hline \# Folders/labels + subfolders & $32(87.51)$ & $8.81(10.10)$ \\
\# Folders/labels with 3 or fewer items & $1.1(2.73)$ & $0.71(1.73)$ \\
\# Folders/labels not used for at least 6 months & $18(66.97)$ & $4(6.23)$
\end{tabular}

Table 1. Average number of folders/labels (with standard deviation), excluding P10 who did not distinguish between work and personal accounts.

for the purpose of this analysis. Not all email clients offer the same features: for example, Gmail does not have folders per se, but its labels can be assigned to the same email, unlike Outlook where an email can only belong to one folder, but can have multiple categories. However, other research also claims that folders and labels are comparable, as their ultimate purpose is to "file messages away from the inbox" ([18], p.797). With regard to the use of different email clients, it is of course possible that other aspects of their design can impact on both management and retrieval strategies which might confound our comparisons of work versus personal email. However, we did not find obvious evidence of this in our sample.

Notably, in our sample, the three healthcare workers (P4, P6, P7) did not have any folders in their work account, but organised their personal email with folders to store important files, such as insurance documents, login details (P7) or emails related to their owned properties (P6). Despite having folders for "important documents" and university-related emails, P4 admitted not having accessed those folders in over a year. This suggests that occupation may play a role in email strategies, as seen in [11], and points towards the idea that for workers who do not rely heavily on email, folders in personal accounts are valuable for long term archiving. However, the findings may not be generalisable to other professions.

\section{What People Looked for in Work vs. Personal Accounts}

From the interviews and diaries, we found that what users looked for differed between work and personal accounts, confirming recent findings [18]. Highlighting how the content differs is relevant for understanding the ways in which they looked for it, given the range and diversity of reasons people need to find things in their accounts.

In personal accounts, participants mainly reported retrieving details and documents such as: order confirmations; travel related information (e.g. booking references); money related email (e.g. bank details, and bills); and to-do lists or reminders. In addition, participants sometimes searched their personal account to go through past correspondence in a more reminiscent or nostalgic way: "A friend of mine (high school) sent me a link to a video on YouTube [...] I was just nostalgic and went to that email again and watched the goal." ( $\mathrm{P} 14$, diary). In work accounts, instead, participants retrieved information which was more central to, and entwined with, ongoing work tasks. Finally, some types of information were reported being looked for in both work and personal accounts: meeting details such as location or time; attachments; links; login details; and pictures. The most frequent reasons for searching either type of account were "double checking" and forwarding information.
In summary, retrieval in work accounts seemed to be more motivated by needing to refer to email in the course of information gathering for larger tasks, as described in [33]. While this can be the case in personal accounts too, retrieval in the latter among our sample tended to involve more directed search for email that contained quick facts (similar to the finding category in [33]).

\section{How People Retrieved Work vs. Personal Email}

Participants were generally good at finding email within one or two attempts, with a $97.5 \%$ success rate from the diary entries and $81 \%$ in the 42 tasks performed during the first interview. When unsuccessful, they still managed to obtain the information needed by other means. For example, they either remembered it was stored somewhere else ("I found the file in my Dropbox" - P11, diary), or asked to have the information sent to them ("So I gave up I said hello to her on WhatsApp and Facebook $"$ " - P15, diary), suggesting that people usually find what they are looking for.

Overall, diary entries showed that participants had preferred retrieval strategies depending on the account: for personal accounts the most common methods were using the search bar and glancing or scrolling the inbox, while for work accounts the most common ones were clicking on a folder and using keyword search within it (folder+search), using the search bar, or simply opening a folder and scrolling through its contents (folder+scroll) (Figure 1). This confirms the heavier reliance, in work email, on the use of structured filing to support retrieval and shows a hybrid combination between preparatory (folder) and opportunistic (search bar/scroll) strategies. It is important to note that strategies depicted in Figure 1 were often used in combination, when the first attempt was not successful, but with no recurring pattern.

Further analysis of the diary entries together with the interview transcripts allowed us to abstract the strategies reported in the diaries and shown in Figure 1. As a result, participants can be described as falling into three different categories:

1. Those whose retrieval strategies referred to only one of their accounts because, despite having multiple accounts, they used only one (P4, P10, P13): "Gmail.com $[\cdots]$ was my personal one but I actually use it for work as well" - P10, who despite having other two accounts for part-time jobs, prefers keeping everything in the same inbox.

2. Those who used similar strategies when retrieving information from both their work and personal accounts (P1, P5, P6, P7, P11, P12, P14, P16): "[I] primarily use the search bar. It's usually order one operation, it's not order N." - P1,

\footnotetext{
$5_{\text {https: } / / \text { www. whatsapp.com }}$

$6_{\text {https: }} / /$ www. facebook.com
} 


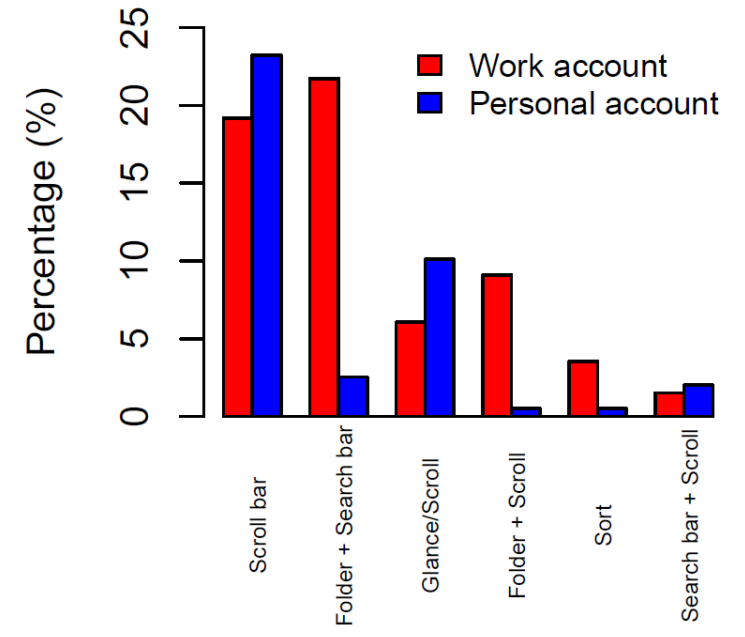

Figure 1. Combined retrieval strategies used for work and personal accounts, as reported in diary entries.

suggesting that he preferred the quickest route for retrieval, with the least number of attempts.

3. Those who used different retrieval strategies for work and personal accounts (P2, P3, P8, P9, P15). While in their personal account they tended to rely on the search bar or scrolling through their inbox, in work accounts they relied more heavily on accessing a folder and then using the search function within it. This is nicely illustrated with comments from P3. Talking about her work account, she said: "[I create folders] to try and group emails that have a common theme together. $[\cdots]$ It's just easier to reference [and] find them if they are altogether somewhere." By contrast, when P3 talked about her personal account, she reported usually just scrolling or using the search bar: "What I tend to do is, if it's really important I don't touch it. But if it is just general crap I would just tend to move it to [archive]."

With regard to the effect of different email clients on patterns of retrieval and management, we could discern no clear relationship between email clients and type of account. While this does not exclude it as a possibility, it is fair to say that differences between work and personal accounts were more salient than any that might be due to particular features of the email client used.

\section{Cross-Account Issues and Workarounds}

In general, despite the overall success reported in diaries of participants finding email, they nonetheless complained about how hard and frustrating it can be, especially considering their many accounts. As a result of this frustration, workarounds were used to guard against these difficulties, including moving away from email altogether.

\section{Making messages conspicuous}

Some participants ensured that the information they needed would be at the top of their inbox, either by using built-in features, such as Save for Later in the Mailbox app "so that it pops up when I need it, which [is] incredibly useful because then it's just at the top of my inbox, it's like 'a-ah!'” (P11, first interview), or repurposing existing features, such as: "I mark important conversations as unread and leave them there in my unread upper-portion of the screen. This way I don't really have to search for them by using the engine" (P15, diary).

\section{Email to self}

Other issues related to retrieving email from oneself. We know from previous literature that self-emails are often used as reminders and to-do lists [2] and as such, they might have a short lifespan until they serve their purpose. However, we found that having to retrieve self-emails can cause issues: "This one it's annoying because $[\cdots]$ it includes me. If I am looking for an email that I sent myself, it also includes an email where I have replied to someone," (P8, first interview). It also created redundant information, "if I send myself a message then I get two copies in the inbox and [in the sent folder]. It's a really silly little thing but it's sort of like kind of irritating" (P11, first interview).

As a result of this, P11 started using Pushbullet ${ }^{7}$ to send himself things, "I can just push this to myself wherever I want it to be and it's kind of, it's more like a dedicated app for it and it feels less messy than sending myself an email, because emails have a lot of sort of metadata and information on it". Pushbullet allows users to share web content such as links with oneself and friends. Other workarounds for searching for self-emails included changing " $m y$ name for myself on my Gmail account to me. And then I just type 'me', it returns ' $m e$ '." (P15, first interview), and creating a folder just for self-emails (P13). In this case, P15 used self-email for short-lived reminders and to-do lists, whereas P13 sent himself emails with long-lived information, e.g. bank details.

\section{Account and channel boundaries}

Another set of issues emerged as a direct result of having multiple email accounts for different purposes. We found that participants experienced difficulties in finding email sometimes because the boundaries between accounts were not welldefined. This affected their filing strategies but also their retrieval behaviour, as this quote exemplifies: "I think the problem arises from the fact that it's personal and work email and sometimes there's tons of overlap $[\cdots]$ so like my sister might send me this, really interesting thing to do with academic things $[\cdots]$ and she might have put like, I don't know, some personal information in there as well about a holiday... I'd be really confused about where to put that and so like, that's why I didn't use the filing system" (P10, first interview).

By contrast, P12 found these concerns were no longer relevant when using Slack ${ }^{8}$, an instant messaging tool advertised for work purposes that allows users to communicate with team members through public channels, private groups and direct messages. As a result, it made it easier for him to retrieve older messages: "Before [my colleague and I] set [Slack] up, it was really horrid, $[\cdots]$ it would all be like, 'Oh, I sent you an email three months ago' or 'I sent you a text' or, like, 'I've got this like WhatsApp chat thing'. But it is all in there and it's

\footnotetext{
7 https://www.pushbullet.com

8 https: //slack.com
} 
all curated heavily and there's no, like, subject changing", in fact, he explains how this is beneficial to maintaining separate work and personal roles, "you're not posting something about going for drinks in the thread about the job we're working on" (P12, first interview).

More generally, we found our younger participants $(n=8$, age 30 ) were moving away from email for personal communications, in favour of other dedicated channels, as has been suggested in popular media ${ }^{9}$. P12 explained this very well, distinguishing the use of email from instant messaging tools and social networks: "I'm not really sure where email sits at the moment $[\cdots]$ not that long ago it was the only option for communicating with people via computers. Now I've got one machine in my hand that can do, like, 50 different types of email sending [‥] I don't use [email] the same way I might use, say, Facebook chat or like texting or like Snapchat." (P12, first interview).

He went on to explain how other people may not be aware of personal preferences, blurring the boundaries not only between work and personal, but also between communication channels, making it more difficult to manage and retrieve information: "People message me on my Facebook to give me a job so I [...] maybe screenshot and email [it to myself], or if someone's emailing me on the wrong account $[\cdots]$ rather than asking them to email me on the proper account $[\cdots]$, I would kind of do it for them. $[\cdots]$ I mean it's putting it where I would end up looking for it" (P12, first interview).

We also found a movement away from email when it came to sharing pictures, choosing different communications channels instead, such as WhatsApp. Participants sent and received photos via email only in two cases: when the sender was not a user of social media or instant messaging services (e.g. sharing with other family members); or when wanting to maintain a certain image quality. "Me and my friend, we are constantly [sharing] pictures or screenshots via WhatsApp. Also Snapchat... But yes, never really on email for social reasons" (P6, first interview).

In summary, we see a shifting landscape where email is finding a new role and niche amongst other channels. The evidence suggests that the difficulty of managing email across account boundaries, along with issues in trying to find information across them, may be contributing factors.

\section{Email Retrieval Across Devices}

Moving on from cross-account issues, we now turn to issues linked to the use of different devices for accessing email. Here we found that $85 \%$ of email retrieval tasks reported in the diaries occurred on laptops and PCs, with only $15 \%$ on mobile devices. More than this, the device used depended on the type of email being sought for or the location of use. Laptops and PCs were used primarily at work and for retrieving work-related content (e.g. correspondence, tasks, instructions), together with important information from personal accounts (e.g. bills, bank details, passport). This is perhaps not surprising given that many work-related tasks, including email,

\footnotetext{
${ }^{9}$ Forbes 2014, http://onforb.es/1s5pT1K
}

require larger screens and keyboards, for example when creating documents.

Although 15 participants accessed at least one of their email accounts on their phone, the majority of them did not search on their mobile devices either because it was not an easy process or they "never had cause to" (P1). Phones were used primarily for searching quick reference information, such as: booking confirmation numbers, meeting details (e.g. location or time), emails to self containing to-do lists (e.g. shopping list). They were also used where other devices were not handy, such as in bed or at a restaurant. Of those who did search on their phone, some had different strategies from their laptops or desktop PCs, as P3 describes: "it's weird, if I am typing it out on the phone I don't think to do [advance search options] but if I was using my Gmail on a laptop I probably would".

Other types of mobile devices (tablets and smartwatches) were not used to search email. Eight participants owned a tablet, and two of them never used it to access email. Tablet use for email was limited and echoes previous findings [18] that suggest tablets are more for information-consumption, such as "for reading eBooks" (P1). While both smartwatch users received email notifications on their wrist, they did not use them for searching. As P1 explains, "the wrist is not the place to search emails".

The relatively little amount of retrieval activity on smartphones despite high engagement with such devices is somewhat surprising. Our interviews revealed that frustration and user unfamiliarity with search features may explain this discrepancy to some extent: "For me it's easier on Outlook [on laptop] but that's probably because I know how to use it better really, because you don't really spend time figuring out how to search for things on your phone" (P16, first interview). Similarly, P6 explains: "I have never really noticed it on my phone and it's my phone that I always check my emails on". Interestingly, we found that not knowing about the search button was not related to technology expertise.

In addition, participants complained about the phone being "less effective" at finding what they were looking for, due to limited storage capabilities or connectivity problems: "I find that on mobile searching is not as good because it's, particularly when you're out and on a $3 G$ connection, it's struggling to find $[\cdots]$ the search heuristic isn't fast enough $[\cdots]$ I sometimes end up with emails that are like from ages ago because it's something that's cached $[\cdots]$ not really relevant to what I'm looking for, just because it's not got enough storage" (P11, first interview).

\section{Cross-Device Issues and Workarounds}

Our findings show how searching email, especially on different devices, can cause frustration: "I've never had particularly good results with searching for emails." (P12, first interview). To avoid this, participants relied on a series of workarounds, especially when anticipating having to retrieve email on the phone and sometimes as a result of the email client.

\section{External copies}

Some of these workarounds were closely linked to general personal information management practices, such as copying 
information onto scraps of paper or into a separate application. For example, P1 used Google Keep ${ }^{10}$, a note-taking app, to keep track of any trip related information: "It's like keeping a single post-it note for it [] so its ephemeral. I mean I don't search in the same way $[\cdots]$ I don't keep an archive of all the post-it notes that are on the fridge, because once that's reached its purpose it's often in the recycle bin" (P1, follow-up interview).

\section{Sending}

Other workarounds included sending oneself an email, a strategy also used across accounts, so to avoid having to search or scroll the inbox when they have already anticipated needing to retrieve an email, as P9 explains: "So say I'm going to [a shop] to pick up an order [...] it's been a few days since I got the email saying the number of the order, then I will forward that email to myself again so that it will be at the top when I get to the shop and I can tell them the number."

\section{Application switch}

Of note, three people did not use their usual email application when they had to search for an email, especially for old ones, as they were not easily accessible from their preferred application. For example, "generally [I use my phone] if I'm out and about, but I will normally go on the web browser and go on Hotmail itself, rather than the app because $\cdots$ on [the Mail app], it only has my most recent ones" (P7, first interview). Mail app has been criticised for its poor searching capabilities ${ }^{11}$ and indeed two of the five Mail app users switched applications when they needed to retrieve a message: P4 switched to the Outlook app on her phone; P7 switched to the webmail on her phone. Other Mail app users did not feel the need to switch application (but some did use other workarounds). This suggests that, as much as email clients do impact user engagement, there are clearly other factors involved.

\section{Pictures and screenshots}

To overcome some of the annoyance with having to search, but particularly the limitations of mobile devices not always having signal, or enough memory to store email, some participants took screenshots of their emails that they could then search for in their photo collection. P7 reported in the diary taking a screenshot of her train confirmation a couple of days before leaving for Paris. She then explained that on her the way back, looking for that screenshot was more difficult, "because I had taken quite a lot of photos on holiday, I did have quite a bit of scrolling to have to do for that one" (P7, follow-up interview). These ephemeral scraps of information were not always deleted from the phone gallery once they served their purpose, adding to the phone's storage problem. This builds on previous work on how scraps of information are needed for rapid capturing [36].

Interestingly, pictures or screenshots were also used to share the content of email with others via different channels, as a means to curate communications: "I will occasionally $[\cdots]$, screenshot an email and send it to him [via WhatsApp] so he

\footnotetext{
$10_{\text {http: //ww . google.com/keep }}$

${ }^{11}$ MacWorld, http://bit.1y/1TI9BWv
}

can deal with it and he doesn't have to be in a thread" (P12, first interview). It is worth mentioning that screenshots and pictures of email were taken by all participants who were in their 20s $(n=8)$ but none of the older participants relied on this workaround.

\section{DISCUSSION}

To the best of our knowledge, this study is the first to provide insights into how people retrieve information across work and personal email accounts, and across devices. By using a mixed method approach, we confirm and extend previous findings, along with presenting several novel insights.

\section{Work versus Personal Email}

We found that work email continues to provide a central archive for work-related tasks, as a key part of knowledge work and in support of structuring tasks, creating documents, and controlling the workflow of tasks. This confirms that email archives continue to be an important hub for working life. Personal email archives, by contrast, are more likely to be searched in order to access important details (e.g. booking references) and documents (e.g. passport). As a result, it is not surprising that work email accounts are searched more often than personal ones because they are in service of these workrelated tasks. Moreover, given that our evidence from younger participants suggests that they are moving away from email as a personal communication channel (preferring other channels instead), we might surmise that in time personal email may well evolve primarily into an archive for what could be called "domestic paperwork".

We also consider retrieval and management practices in light of participants' email clients, extending previous work [18]. We find that participants using the same clients had different experiences and the choice of email client alone does not explain issues encountered and workarounds created: personal preference and type of account also play a significant role. Further work should expand on this by including a larger sample.

We find some points of contrast with earlier work related to how people retrieve email in the context of their management practices. For example, whereas previous work [18] found similar management habits for work and personal accounts, we found that work accounts were usually more structured, with more folders than personal accounts. This partially confirms what Whittaker et al. [38] concluded, that folders are created as a way of managing the increasing number of messages, at least in work accounts. In personal accounts, instead, the small number of folders confirms how users may not want to put effort into organising information [5]. However, we did also see how some participants relied on additional accounts to filter out unwanted messages from their main accounts.

These differences in management strategies were also reflected in different retrieval strategies between work and personal accounts. Table 1 and Figure 1 provide a good summary that highlights the trade-off between preparatory and opportunistic mechanisms [38]: we found that folders in work accounts were used for later retrieval, as opposed to in personal accounts where opportunistic retrieval was more common due to 
the lack of a filing system. While the overall most common retrieval strategy was using the search bar, work accounts were primarily searched starting with a folder, and then searching or scrolling within it, thus combining preparatory (folder) and opportunistic (search, scroll) mechanisms. This means that the two strategies are not mutually exclusive, as was suggested by [38] but rather represent a kind of hybrid approach.

\section{Management Through Multiple Channels}

Another set of findings take us outside of the conventional email ecosystem to look for other ways in which email was managed. For example, there is a trend toward new kinds of dedicated work-related tools that are becoming popular, such as Slack ${ }^{8}$ and Asana ${ }^{12}$, among others, which incorporate email-like functionality. Slack is an instant messaging (IM) tool advertised for work purposes that allows users to communicate and share files with team members through public channels, private groups and direct messages. Asana, too, offers team collaboration functionalities that allows users to communicate, share, and monitor progress on individual projects, with the purpose of moving away from email. In addition, both tools offer the possibility to integrate a wide range of other applications, such as Dropbox ${ }^{13}$ and Evernote ${ }^{14}$, supporting users' flexibility and preferences by creating a tailored tool. In essence, what we are seeing here might be described as a trend towards greater curation of content for particular purposes, which also serves as a way of managing it for later retrieval, which tools such as Slack, Asana and others afford.

Another way of viewing this is that using other channels to transfer information, communicate, share pictures, and store data, is a way for users to step away from having to rely on email retrieval, which they find frustrating and not always efficient. Our younger participants in particular were more aware of other communication channels and articulated more frustration with the current limitations of email systems. By contrast, people who were heavier users of email appeared more willing to put up with the limitations perhaps because they had not been exposed to alternatives.

We might surmise, however, that using ever more channels also exacerbates both managing and retrieving information sent between people. Indeed we saw this as a result of trying to deal with multiple email accounts where participants often struggled to remember from which account an email was sent or where it was received, a problem that was most prevalent when the cross-over happened between work and personal accounts. This highlights the fact that the boundaries between accounts are not always well defined, both in terms of how a user thinks of them and how others perceive them. This was despite the fact that, by having multiple accounts, clearly reflecting different life roles (personal, professional, social, etc.), at least some participants were striving to draw those boundaries to make management and retrieval easier.

To the best of our knowledge no work has looked at the use of these new kinds of tools, particularly comparing them with

\footnotetext{
12 https : //asana.com

13 https : //www.dropbox.com

14 https: //evernote.com
}

email. Nor has it examined the added benefits or difficulties that might ensue by using more and different channels to take over from email. While our findings point to why these new tools are becoming popular, more research is needed to explore these issues in depth and to examine their consequences.

\section{Management Through Workarounds}

We have seen that both filing strategies as well as the use of multiple channels or accounts have important implications for how email is retrieved. However, taking a cross-device view also highlights other strategies that users take in order to anticipate the need for later retrieval. We saw this particularly in the case of mobile devices, where our participants avoided the need to search for email as much as possible. In part, this was because of storage and connectivity issues; in part, this was due to a perception that search tools on mobile devices are ineffective and difficult to use. As a consequence, our participants used a variety of workaround strategies. For example they took screenshots of booking references, or transferred information through dedicated apps for easier and quicker access to information when needed. Indeed, such workarounds which made certain email messages more conspicuous and easily accessed could also be seen within accounts, such as the practice of highlighting email in the inbox. This seems symptomatic of the difficulty of quickly finding the information one is after. We also found that other mobile devices, like tablets and smartwatches, were not used for searching email. Such findings, although more anecdotal than anything, are interesting as new research is considering how smartwatches are being used [10].

\section{CONSIDERATIONS FOR DESIGN}

Whilst our findings are not representative of the whole population, they do provide useful insights on current retrieval practices in today's email ecosystem which is increasingly one based on multiple accounts and multiple devices. While aspects of these behaviours will continue to change with technology, we believe that the themes and insights from our data provide useful pointers on how email clients could be re-designed, or email functionality could fit within the larger scope of new applications and services. Some suggestions are as follows:

- Better email integration for work. Our findings suggest that work and personal email both serve important archiving functions, but in different ways. Work email archives are tied more closely with knowledge work tasks and hence communication functions could be better integrated into authoring and workflow tools. More than this, however, the findings suggest that email as an archive is equally important, but when it comes to work-related email, the fractionation of multiple accounts and devices creates additional silos that complicate management and finding. We propose that design should aim to counteract this trend and find more opportunities to tie together email archives with work resources, as well as binding across accounts and devices.

- Learning from other communication tools. As popular IM tools take over not only in personal, but also for work communications, email clients could, and perhaps should, 
learn from such tools. For example, Microsoft recently announced Send ${ }^{15}$, a mobile app that allows sending quick messages masked as work emails (similar to Gmail Hangout conversations ${ }^{16}$ ). However, these systems are still unlinked to email content and cannot be easily searched. They also add to the problem of managing different communication channels. Tools such as Slack, instead, nudge users to curate content in a more easily retrievable way, but the move toward quick, more synchronous communication may change the nature of work in other ways. More work is needed to explore the benefits and drawbacks of IM in the workplace.

- Designing for short-lived and long-lived content. Changing our focus from work email, personal email teaches us that email could be imbued with more features which allow paperwork, forms, and facts to be accessed and filed, either for quick access or long term storage. With regard to quick access, strategies that make email content quickly accessible, but then obsolete once used, suggest that it might be useful to look for ways to automatically classify them as such. Self-email largely falls into the category of being utility based, but only useful in the short term. We need to think about mechanisms for making such information conspicuous but temporary. Likewise, some emailed documents such as warranties, instructions, and visas are more likely to be needed long term but can be kept in "cold storage". There is no need for visibility, rather the emphasis is on safe-keeping and back-up. This points to more diverse options for storing and archiving information sent by email.

- Optimising smartphone search. Finally, it is clear from our findings that search on mobile devices needs to be improved. While storage and connectivity may be less of an issue in the future, we urge designers to learn from the workarounds we presented. For example, searching on mobiles could be enhanced by allowing users to pin emails from any device in a separate application or to the lock screen, thus providing a new feature to quickly refer to ephemeral scraps of information[1]. By moving outside of the inbox, this would also avoid messages on the top being hidden by new incoming emails, and bypass connectivity or storage issues.

\section{CONCLUSIONS}

In this paper we present findings from an in-depth qualitative field study which takes a new look at how email management and retrieval occurs in today's multi-account, multi-device world. The findings point to how users' attempts to manage their email through a proliferation of accounts comes with its own problems: different accounts are used for different purposes, the boundaries between accounts can be blurred, and the mechanisms and strategies for managing and retrieving are account-dependent. Likewise, it is clearly important that we have access to and engage with email through a variety of devices, yet when it comes down to it, finding email on handheld devices such as phones is still seen as difficult. Some of this is doubtless due to the need to intertwine work email with the tools of work which mainly reside on the laptop and

\footnotetext{
$15_{\text {http: //bit.1y/1CSOAGT }}$

$16_{\text {https }} / / /$ hangouts .google.com
}

PC, though we have also pointed to other limitations in the way mobile devices are designed. In all of this, we suggest that one way forward is to think about new features for email tools based on these findings. In the longer term, however, we may well see email evolve not as a stand-alone application, but as a tightly integrated set of communication and archiving functions within more flexible applications and services.

\section{REFERENCES}

1. Deborah Barreau and Bonnie A Nardi. 1995. Finding and reminding: file organization from the desktop. ACM SigChi Bulletin 27, 3 (1995), 39-43.

2. Victoria Bellotti, Nicolas Ducheneaut, Mark Howard, and Ian Smith. 2003. Taking email to task: the design and evaluation of a task management centered email tool. In Proceedings of the SIGCHI conference on Human factors in computing systems. ACM, 345-352.

3. Ofer Bergman, Maskit Tene-Rubinstein, and Jonathan Shalom. 2013. The use of attention resources in navigation versus search. Personal and ubiquitous computing 17, 3 (2013), 583-590.

4. Richard Boardman and M Angela Sasse. 2004. Stuff goes into the computer and doesn't come out: a cross-tool study of personal information management. In Proceedings of the SIGCHI conference on Human factors in computing systems. ACM, 583-590.

5. Olha Bondarenko and Ruud Janssen. 2005. Documents at hand: Learning from paper to improve digital technologies. In Proceedings of the SIGCHI conference on Human factors in computing systems. ACM, 121-130.

6. Virginia Braun and Victoria Clarke. 2006. Using thematic analysis in psychology. Qualitative research in psychology 3, 2 (2006), 77-101.

7. Robert Capra, Julia Khanova, and Sarah Ramdeen. 2013. Work and personal e-mail use by university employees: PIM practices across domain boundaries. Journal of the American Society for Information Science and Technology 64, 5 (2013), 1029-1044.

8. Juan Pablo Carrascal and Karen Church. 2015. An in-situ study of mobile app \& mobile search interactions. In Proceedings of CHI, Vol. 15. 2739-2748.

9. Scott Carter and Jennifer Mankoff. 2005. When participants do the capturing: the role of media in diary studies. In Proceedings of the SIGCHI conference on Human factors in computing systems. ACM, 899-908.

10. Marta E Cecchinato, Anna L Cox, and Jon Bird. 2015a. Smartwatches: the Good, the Bad and the Ugly?. In Proceedings of the 33rd Annual ACM Conference Extended Abstracts on Human Factors in Computing Systems. ACM, 2133-2138.

11. Marta E Cecchinato, Anna L Cox, and Jon Bird. 2015b. Working 9-5?: Professional Differences in Email and Boundary Management Practices. In Proceedings of the 33rd Annual ACM Conference on Human Factors in Computing Systems. ACM, 3989-3998. 
12. Edward Cutrell, Susan T Dumais, and Jaime Teevan. 2006a. Searching to eliminate personal information management. Commun. ACM 49, 1 (2006), 58-64.

13. Edward Cutrell, Daniel Robbins, Susan Dumais, and Raman Sarin. 2006b. Fast, flexible filtering with phlat. In Proceedings of the SIGCHI conference on Human Factors in computing systems. ACM, 261-270.

14. David Dearman and Jeffery S Pierce. 2008. It's on my other computer!: computing with multiple devices. In Proceedings of the SIGCHI Conference on Human factors in Computing Systems. ACM, 767-776.

15. Nicolas Ducheneaut and Victoria Bellotti. 2001. E-mail as habitat: an exploration of embedded personal information management. interactions 8, 5 (2001), 30-38.

16. Susan Dumais, Edward Cutrell, Jonathan J Cadiz, Gavin Jancke, Raman Sarin, and Daniel C Robbins. 2003. Stuff I've seen: a system for personal information retrieval and re-use. In Proceedings of the 26th annual international ACM SIGIR conference on Research and development in informaion retrieval. ACM, 72-79.

17. Danyel Fisher, AJ Brush, Eric Gleave, and Marc A Smith. 2006. Revisiting Whittaker \& Sidner's email overload ten years later. In Proceedings of the 200620 th anniversary conference on Computer supported cooperative work. ACM, 309-312.

18. Catherine Grevet, David Choi, Debra Kumar, and Eric Gilbert. 2014. Overload is overloaded: email in the age of Gmail. In Proceedings of the SIGCHI Conference on Human Factors in Computing Systems. ACM, 793-802.

19. Radicati Group. 2015. Email Statistics Report, 2015-2019. (2015).

20. Jacek Gwizdka. 2002. Reinventing the inbox: supporting the management of pending tasks in email. In CHI'O2 Extended Abstracts on Human Factors in Computing Systems. ACM, 550-551.

21. Benjamin V Hanrahan, Manuel A Pérez-Quiñones, and David Martin. 2014. Attending to Email. Interacting with Computers (2014), iwu048.

22. Daniel Harrison, Paul Marshall, Nadia Bianchi-Berthouze, and Jon Bird. 2015. Activity tracking: barriers, workarounds and customisation. In Proceedings of the 2015 ACM International Joint Conference on Pervasive and Ubiquitous Computing. ACM, 617-621.

23. Tero Jokela, Jarno Ojala, and Thomas Olsson. 2015. A Diary Study on Combining Multiple Information Devices in Everyday Activities and Tasks. In Proceedings of the 33rd Annual ACM Conference on Human Factors in Computing Systems. ACM, 3903-3912.

24. Amy K Karlson, Brian R Meyers, Andy Jacobs, Paul Johns, and Shaun K Kane. 2009. Working overtime: Patterns of smartphone and PC usage in the day of an information worker. In Pervasive computing. Springer, 398-405.
25. Fahim Kawsar and AJ Brush. 2013. Home computing unplugged: why, where and when people use different connected devices at home. In Proceedings of the 2013 ACM international joint conference on Pervasive and ubiquitous computing. ACM, 627-636.

26. Wendy E Mackay. 1988. Diversity in the use of electronic mail: A preliminary inquiry. ACM Transactions on Information Systems (TOIS) 6, 4 (1988), 380-397.

27. Tara Matthews, Jeffrey Pierce, and John Tang. 2009. No smart phone is an island: The impact of places, situations, and other devices on smart phone use. IBM RJ10452 (2009).

28. George D Montañez, Ryen W White, and Xiao Huang. 2014. Cross-device search. In Proceedings of the 23rd ACM International Conference on Conference on Information and Knowledge Management. ACM, 1669-1678.

29. Antti Oulasvirta and Lauri Sumari. 2007. Mobile kits and laptop trays: managing multiple devices in mobile information work. In Proceedings of the SIGCHI conference on Human factors in computing systems. ACM, 1127-1136.

30. Pew Research. 2014. Device Ownership. (2014).

31. Stephanie Santosa and Daniel Wigdor. 2013. A field study of multi-device workflows in distributed workspaces. In Proceedings of the 2013 ACM international joint conference on Pervasive and ubiquitous computing. ACM, 63-72.

32. Abigail J Sellen and Richard HR Harper. 2002. The myth of the paperless office. MIT press.

33. Abigail J Sellen, Rachel Murphy, and Kate L Shaw. 2002. How knowledge workers use the web. In Proceedings of the SIGCHI conference on Human factors in computing systems. ACM, 227-234.

34. Hilary Smith, Yvonne Rogers, and Mia Underwood. 2003. Managing personal and work email in the same box: overcoming the tensions through new metaphors. Proc. HOIT 2003 (2003).

35. Juha Takkinen and Nahid Shahmehri. 1998. Delegation of tasks and dissemination of information in organizations: Restructuring internet e-mail for doing things. AMCIS 1998 Proceedings (1998), 166.

36. Max G Van Kleek, Michael Bernstein, Katrina Panovich, Gregory G Vargas, David R Karger, and mc schraefel. 2009. Note to self: examining personal information keeping in a lightweight note-taking tool. In Proceedings of the SIGCHI Conference on Human Factors in Computing Systems. ACM, 1477-1480.

37. Steve Whittaker, Victoria Bellotti, and Jacek Gwizdka. 2006. Email in personal information management. Commun. ACM 49, 1 (2006), 68-73. 
38. Steve Whittaker, Tara Matthews, Julian Cerruti, Hernan Badenes, and John Tang. 2011. Am I wasting my time organizing email?: a study of email refinding. In Proceedings of the SIGCHI Conference on Human Factors in Computing Systems. ACM, 3449-3458.

39. Steve Whittaker and Candace Sidner. 1996. Email overload: exploring personal information management of email. In Proceedings of the SIGCHI conference on Human factors in computing systems. ACM, 276-283. 